Research Article

\title{
The Association between Plasma Levels of Trimethylamine N-Oxide and the Risk of Coronary Heart Disease in Chinese Patients with or without Type 2 Diabetes Mellitus
}

\author{
Zengxiang Dong, ${ }^{1,2}$ Zhaoguang Liang, ${ }^{2}$ Meihua Guo, ${ }^{1}$ Shuang Hu, ${ }^{1}$ Zhaoqian Shen, ${ }^{2}$ \\ and Xin Hai $\mathbb{D}^{1}$ \\ ${ }^{1}$ Department of Pharmacy, The First Affiliated Hospital of Harbin Medical University, Harbin 150001, China \\ ${ }^{2}$ Department of Cardiology, The First Affiliated Hospital of Harbin Medical University, Harbin 150001, China \\ Correspondence should be addressed to Xin Hai; hai_xin@163.com
}

Received 23 May 2018; Accepted 12 July 2018; Published 8 August 2018

Academic Editor: Donald H. Chace

Copyright (c) 2018 Zengxiang Dong et al. This is an open access article distributed under the Creative Commons Attribution License, which permits unrestricted use, distribution, and reproduction in any medium, provided the original work is properly cited.

\begin{abstract}
Aim. Trimethylamine N-oxide (TMAO) has been demonstrated as an independent risk factor for cardiovascular disease. Our objective was to determine the plasma levels of TMAO in Chinese coronary heart disease (CHD) patients with or without type 2 diabetes mellitus (T2DM). Methods. A total of 132 control participants, 243 CHD patients, and 175 CHD patients with T2DM were enrolled. Plasma levels of TMAO in all patients were measured and analyzed. Results. The plasma levels of TMAO were significantly higher in CHD patients than in control subjects $(3.08 \pm 0.13 \mu \mathrm{M}$ versus $1.49 \pm 0.05 \mu \mathrm{M} ; P<0.01)$. In addition, plasma levels of TMAO were remarkably increased in CHD patients with T2DM compared with CHD patients $(7.63 \pm 0.97 \mu \mathrm{M}$ versus $3.08 \pm 0.13 \mu \mathrm{M} ; P<0.01)$. The receiver operating characteristic analysis revealed that the area under the curve of TMAO was 0.794 and 0.927 to predict CHD or CHD-T2DM patients $(P<0.01)$. Univariate and multivariate logistic regression analysis showed that TMAO was an independent predictor in CHD patients with or without T2DM. The level of TMAO was correlated with high-sensitive troponin I (hs-TnI) and creatine kinase MB (CKMB). Conclusions. TMAO was an independent predictor of CHD in Chinese patients; moreover, the TMAO levels were highly associated with diabetes in CHD patients.
\end{abstract}

\section{Introduction}

Coronary heart disease (CHD) is a major cause of morbidity and mortality in people with diabetes mellitus. Type 2 diabetes mellitus (T2DM) is a metabolic disorder characterized by systemic insulin resistance, which promotes hyperglycemia [1], and it has been found that these metabolic abnormalities lead to increased risk of cardiovascular diseases. So far, the occurrence and development of CHD in diabetic patients are complex and not fully understood [2]. The clinical importance of identifying CHD-T2DM risk is that it can contribute to the determination of the treatment and prevention method of the disease. When both CHD and T2DM occur, a comprehensive prevention of cardiovascular risk factors should be performed in order to reduce the morbidity and mortality of the disease. Rather than simply lowering blood glucose or blood pressure, the comprehensive treatment strategy should include correcting abnormity of blood lipid, glucose, and pressure, reasonable exercise, weight control, and cessation of smoking. In particular, many studies have indicated that diet pattern plays an important role in CHD-T2DM risk $[3,4]$.

Trimethylamine $\mathrm{N}$-oxide (TMAO) is a plasma metabolite from nutrient precursors (choline, phosphatidylcholine, and L-carnitine), which is produced by gut microbiota. Many studies indicated the participation of gut microbes in the pathogenesis of cardiovascular disease [5-8]. Furthermore, numerous studies have revealed a relation between plasma levels of TMAO and cardiovascular risks [9-17]. These studies have indicated that elevated plasma levels of TMAO were each associated with incident risk of major adverse cardiac events independent of traditional risk factors [9], 
TABLE 1: Baseline characteristics of the patients.

\begin{tabular}{|c|c|c|c|}
\hline Characteristic & Control $(n=132)$ & $\mathrm{CHD}(n=243)$ & CHD-T2DM $(n=175)$ \\
\hline Age (years) & $61.9 \pm 14.8$ & $61.8 \pm 11.1$ & $64.4 \pm 10.9$ \\
\hline Male (\%) & $48(36.4 \%)$ & $109(44.9 \%)$ & $76(43.4 \%)$ \\
\hline Hypertension (\%) & $37(28.0 \%)$ & $129(53.1 \%)^{*}$ & $118(67.4 \%)^{* \#}$ \\
\hline Current smoking (\%) & $3(2.3 \%)$ & $20(8.2 \%)^{*}$ & $19(10.9 \%)^{* \#}$ \\
\hline Alcohol intake (\%) & $42(31.8 \%)$ & $67(27.6 \%)$ & $59(33.7 \%)$ \\
\hline $\mathrm{HDL}(\mathrm{mmol} / \mathrm{L})$ & $1.30 \pm 0.03$ & $1.29 \pm 0.02$ & $1.18 \pm 0.02$ \\
\hline $\mathrm{LDL}(\mathrm{mmol} / \mathrm{L})$ & $2.82 \pm 0.06$ & $2.88 \pm 0.05$ & $2.79 \pm 0.06$ \\
\hline Total cholesterol (mmol/L) & $4.73 \pm 0.09$ & $4.79 \pm 0.07$ & $4.60 \pm 0.10$ \\
\hline Triglyceride (mmol/L) & $1.72 \pm 0.09$ & $1.79 \pm 0.07$ & $2.23 \pm 0.12$ \\
\hline Lipoprotein (a) (g/L) & $1.28 \pm 0.02$ & $1.26 \pm 0.02$ & $1.21 \pm 0.02$ \\
\hline Lipoprotein (b) (g/L) & $0.98 \pm 0.03$ & $1.02 \pm 0.02$ & $1.04 \pm 0.03$ \\
\hline Blood glucose $(\mathrm{mmol} / \mathrm{L})$ & $5.32 \pm 0.15$ & $5.33 \pm 0.09$ & $8.65 \pm 0.29^{* \#}$ \\
\hline hs-TnI (ng/mL) & $0.012 \pm 0.003$ & $0.253 \pm 0.104^{*}$ & $0.251 \pm 0.006^{*}$ \\
\hline CKMB (ng/mL) & $1.01 \pm 0.07$ & $2.35 \pm 0.64^{*}$ & $2.97 \pm 0.61^{*}$ \\
\hline
\end{tabular}

Data are presented as mean \pm standard deviation or proportions. HDL: high-density lipoprotein; LDL: low-density lipoprotein; hs-TnI: high-sensitive troponin I; CKMB: creatine kinase MB. ${ }^{*} P<0.05$ versus control; ${ }^{\#} P<0.05$ versus CHD.

some of which found that high TMAO levels portended higher long-term adverse clinical outcomes in patients with heart failure $[10,11]$, and plasma levels of TMAO were an independent predictor of a high atherosclerotic burden in patients with CHD [15]. Moreover, a study has found that diabetes was associated with higher TMAO plasma levels [14]. However, the further relationship between TMAO levels and risks for CHD in patients with T2DM has not yet been examined. A rational hypothesis is thus that circulating levels of TMAO could predict and induce incident risks for CHD among subjects presenting with T2DM, which could provide new idea and method for the prevention of CHDT2DM. Herein, we sought to explore the relationship between plasma levels of TMAO and CHD among patients with T2DM.

\section{Methods}

2.1. Participants. Between May 2016 and August 2017, 550 patients presented to the First Affiliated Hospital of the Harbin Medical University (Harbin, China). The study consisted of three subject groups: control subjects $(n=132)$, CHD patients $(n=243)$, and CHD patients with T2DM $(n=175)$. The clinical characteristics of the study population are summarized in Table 1.

2.2. Ethical Approval of Studies and Informed Consent. The study protocols and the procedures for handling human samples were approved by the Ethics Committee of the First Affiliated Hospital of Harbin Medical University (Harbin, China), and all patients gave informed consent. All the methods were carried out in accordance with the approved guidelines.

2.3. Collection and Handling of Human Blood Samples. Whole blood samples ( $1 \mathrm{~mL}$ per patient) were drawn from the study subjects via a direct venous puncture into the tubes containing EDTA. The human whole blood samples in vacuum tubes were kept at $4^{\circ} \mathrm{C}$ and then centrifuged at $765 \mathrm{~g}$ (centrifuge: Allegra 64R, Beckman Coulter, USA; rotor: Beckman F1010, USA) for $15 \mathrm{~min}$ at $4^{\circ} \mathrm{C}$ to obtain plasma samples.

2.4. Determination of TMAO by LC-MS/MS. We measured TMAO by LC-MS/MS using $50 \mu \mathrm{M}$ TMAO-d9 (TRC, Canada) as an internal standard according to the previous method [17]. Plasma was deproteinized by a mixture with an internal standard solution and methanol (19:1:60 vol:vol:vol). $5 \mu \mathrm{L}$ of each sample supernatant was injected in an Agilent 1100 high-performance liquid chromatography system (Agilent Technologies, USA), and analytes were separated on a Phenomenex Luna Silica column $(100 \mathrm{~mm} \times 2 \mathrm{~mm}, 3 \mu \mathrm{m}$ particle size $)$ protected by a guard column $(4 \mathrm{~mm} \times 2 \mathrm{~mm}$ silica filter $)$ at room temperature. The mobile phase consisted of $88 \%$ methanol containing $10 \mathrm{mM}$ ammonium formate and $0.2 \%$ formic acid $(v / v)$ and $12 \%$ of $10 \mathrm{mM}$ ammonium formate in water containing $0.2 \%$ formic acid $(v / v)$ at a flow rate of $0.2 \mathrm{~mL} / \mathrm{min}$. MS was performed on an API 4000 triple quadrupole mass spectrometer (AB Sciex, USA) with electrospray ionization in the positive mode. Ion transitions used for quantitation were $\mathrm{m} / \mathrm{z} 76 \rightarrow 58$ for TMAO and $\mathrm{m} / z \quad 85 \rightarrow 66$ for the internal standard.

2.5. Statistical Analysis. Continuous variables were described as mean $\pm \mathrm{SD}$ (standard deviation). Categorical variables were presented as the percentage of total patients. Differences between groups in continuous variables and in categorical variables were analyzed by the unpaired 2-tailed $t$-test and by the chi-squared test, respectively. Univariate and multivariate logistic regression analyses were used to assess the association between the risk of TMAO and CHD/CHDT2DM patients. Odds ratios (OR) were presented with 95\% confidence intervals (CI) to show the risk of an event when 
a factor was apparent. To evaluate the levels of TMAO to predict $\mathrm{CHD} / \mathrm{CHD}-\mathrm{T} 2 \mathrm{DM}$ patients, the receiver operating characteristic (ROC) curves were constructed and the area under the curves (AUC) were calculated. The correlation coefficient for the association of TMAO with conventional prognostic markers of CHD patients was measured by Pearson's correlation analysis. All analyses were carried out with SPSS v17.0 software. Statistical significance was accepted at $P<0.05$ for all analyses.

\section{Result}

3.1. Clinical Characteristics of the Study Population. The study consisted of three subject groups: 132 control subjects, 243 CHD patients, and 175 CHD patients with T2DM. Table 1 shows the clinical and demographic characteristics of the patients enrolled in this study. In all the subject groups, there was no statistically significant difference in the age and sex distribution $(P>0.05)$, whereas high-sensitive troponin I (hs-TnI) and creatine kinase MB (CKMB) levels differed significantly between CHD patients with or without T2DM and control $(P<0.05)$.

3.2. Plasma Levels of TMAO in CHD and CHD-T2DM Patients. LC-MS/MS analysis was used to determine the concentration of TMAO in plasma. As illustrated in Figure 1, TMAO demonstrated significant differences in plasma samples between control, CHD, and CHD-T2DM. The levels of circulating TMAO were significantly higher in $\mathrm{CHD}$ patients than in control subjects $(3.08 \pm 0.13 \mu \mathrm{M}$ versus $1.49 \pm 0.05 \mu \mathrm{M} ; P<0.01)$. In addition, plasma levels of TMAO were remarkably increased in CHD patients with T2DM compared with control $(7.63 \pm 0.97 \mu \mathrm{M}$ versus $1.49 \pm 0.05 \mu \mathrm{M} ; P<0.01)$ or CHD patients $(7.63 \pm 0.97 \mu \mathrm{M}$ versus $3.08 \pm 0.13 \mu \mathrm{M} ; P<0.01)$.

3.3. Evaluation of TMAO as a New Biomarker for CHD and CHD-T2DM. According to the established fact that TMAO present in plasma levels were significantly altered in CHD/ CHD-T2DM patients, we sought to determine the potential utility of circulating TMAO as a diagnostic biomarker of CHD and CHD-T2DM. For this purpose, ROC analysis was performed to evaluate the predictive power of circulating TMAO for control, CHD, and CHD-T2DM. Our results showed that the area under ROC curve of TMAO was 0.794 (95\% CI $=0.751 \sim 0.838)$ for $\mathrm{CHD}$ and $0.927(95 \%$ $\mathrm{CI}=0.899 \sim 0.954$ ) for CHD-T2DM (Figures 2(a) and 2(b)). Meantime, we used the area under ROC curve to evaluate the predictive power of circulating TMAO levels and blood glucose levels for CHD-T2DM. Our results showed that the area under ROC curve of TMAO was 0.697 (95\% CI = $0.646 \sim 0.747$ ) for CHD-T2DM and the area under ROC curve of blood glucose was 0.894 (95\% CI $=0.859 \sim 0.930)$ for CHD-T2DM (Figures 2(c) and 2(d)).

The univariate analysis with logistic regression showed that the odds ratio (OR) value was 3.621 (95\% CI: 2.581 5.080) for TMAO $(P<0.01)$ between control and CHD and the OR value was 9.581 (95\% CI: 5.451 16.840) for TMAO $(P<0.01)$ between control and CHD-T2DM

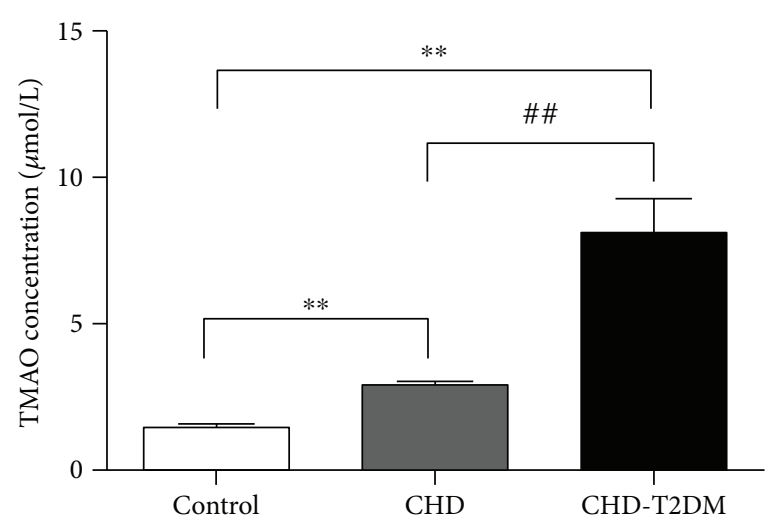

Figure 1: The plasma levels of TMAO. TMAO levels were confirmed in control, CHD, and CHD-T2DM, respectively. ${ }^{* *} \mathrm{P}<$ 0.01 versus control group, ${ }^{\#} P<0.01$ versus $C H D$ group.

(Table 2). The multivariate logistic regression analysis further verified TMAO as an independent predictor for CHDT2DM: the OR value was 3.469 (95\% CI: 2.294 5.245, $P<$ $0.01)$ for TMAO between control and CHD and the OR value was $9.110(95 \% \mathrm{CI}: 4.427 \sim 18.750, P<0.01)$ for TMAO between control and CHD-T2DM (Table 3).

3.4. Relation of TMAO to Conventional Prognostic Markers of CHD. The risk of CHD can be estimated by determining the hs-TnI and CKMB. To further evaluate the usefulness of circulating TMAO as a CHD or CHD-T2DM biomarker, we tested whether the level of TMAO was correlated with conventional prognostic markers of CHD. The data summarized in Table 4 shows that TMAO is positively correlated with hs-TnI and CKMB in CHD and CHD-T2DM.

\section{Discussion}

The present study provides new information about the relation between plasma levels of TMAO and CHD/CHDT2DM in Chinese patients. It had been reported that TMAO was a significant risk factor of cardiovascular disease, which had been demonstrated as an independent predictor of a high atherosclerotic burden and heart failure in patients $[10,15]$. Although these previous studies consisted of large numbers of patients, there was no study focusing on the relation between TMAO and Chinese CHD/CHD-T2DM patients. We demonstrated that the TMAO was an independent predictor of $\mathrm{CHD}$ beyond the traditional prognostic markers in CHD patients. The further information of TMAO could provide an incremental predictive value for the risk of CHD-T2DM, over the traditional prognostic markers, which was based on statistical analysis of clinical variables.

Recently, CHD still remains the leading cause of death worldwide [18-20] and is also a major cause of morbidity and mortality in people with T2DM [3]. In order to improve outcomes of patients with cardiovascular disease, many studies have focused on novel modifiable risk factors, with a special attention to environmental sources $[21,22]$. Although diet has always been linked to outcomes of cardiovascular disease, the important role of gut microbiota has only 


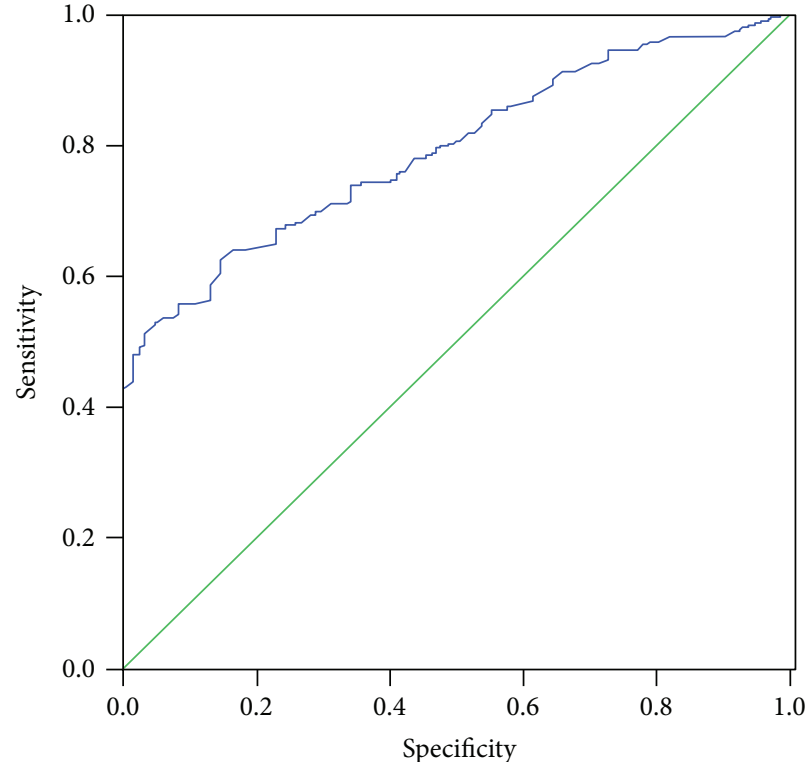

(a)

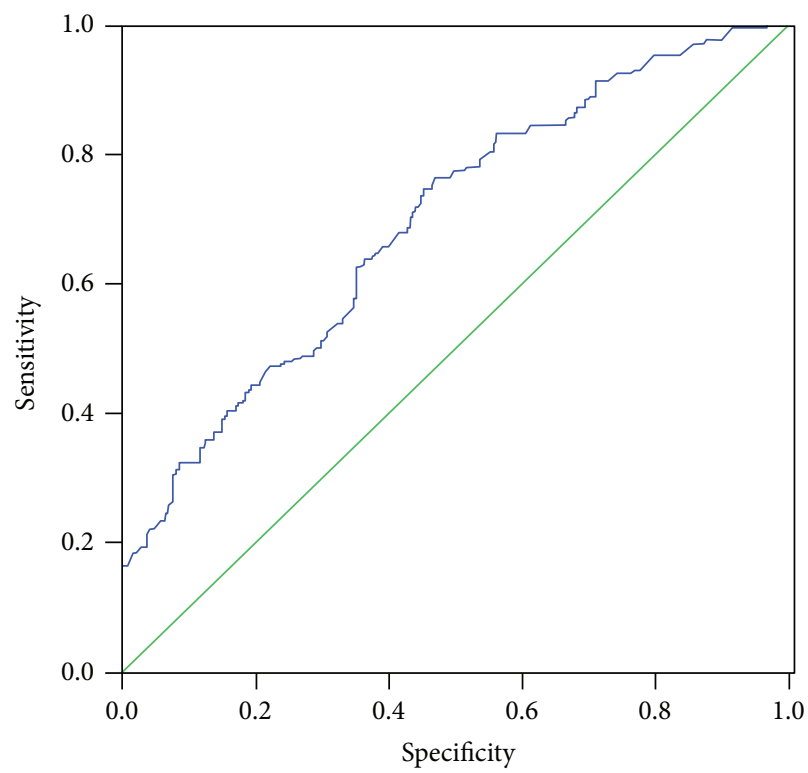

(c)

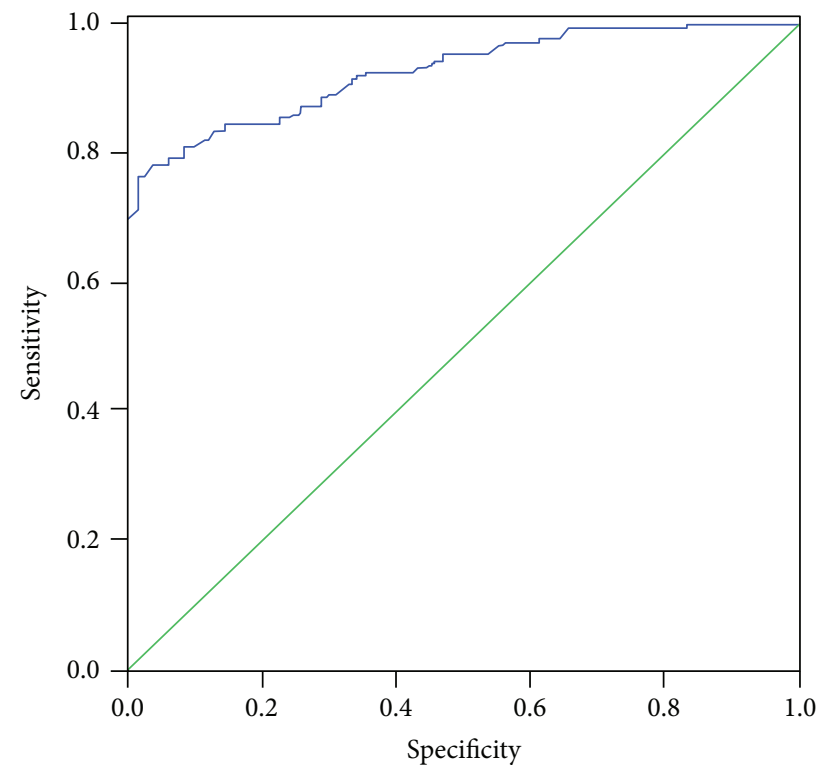

(b)

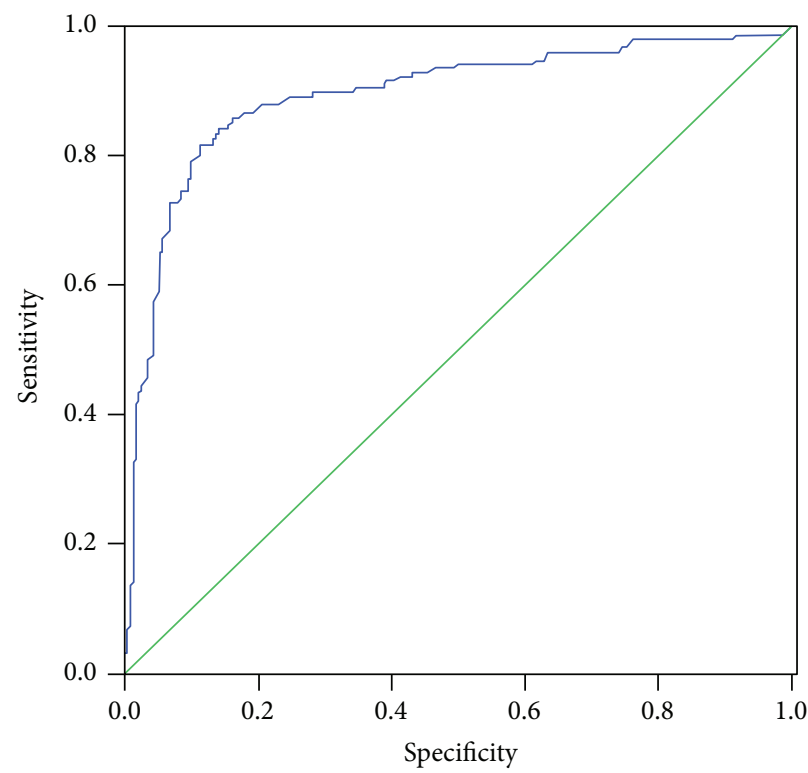

(d)

Figure 2: Receiver operator characteristic analysis of TMAO for predicting CHD and CHD-T2DM. The area under ROC curve was determined to evaluate the predictive power of circulating TMAO levels for CHD (a) and CHD-T2DM (b). The area under ROC curve was determined to evaluate the predictive power of circulating TMAO levels (c) and blood glucose levels (d) for CHD-T2DM.

recently been recognized in the field of cardiovascular disease. Hence, we have sought to research the impact of the gut microbiota-dependent metabolite, TMAO, on CHD in Chinese patients with or without T2DM. Our study demonstrated that circulating TMAO levels were elevated in both patients with CHD and CHD-T2DM. Another novel finding in our results is that circulating TMAO was able to discriminate T2DM patients with CHD from CHD patients and control subjects with an area under the curve of the ROC of 0.927 (95\% CI $=0.899 \sim 0.954)$ and 0.794 (95\% $\mathrm{CI}=0.751 \sim 0.838)$, respectively, which suggested that circulating TMAO exhibited not only a potential biomarker for
CHD but also a biomarker for predicting CHD patients with T2DM. Our results were further confirmed in univariate regression analysis and multivariate logistic regression analysis which revealed strong association of TMAO expression with $\mathrm{CHD} / \mathrm{CHD}-\mathrm{T} 2 \mathrm{DM}$. We also showed that circulating TMAO was significantly and positively correlated with hs-TnI and CKMB in CHD/CHD-T2DM, as determined using Pearson's correlation analysis.

A recent study suggests that plasma levels of TMAO among patients with chest pain may be a risk predictor of incident cardiovascular events and may provide clinical benefit in risk stratification among patients with acute 
TABLE 2: Univariate regression analysis for the association of TMAO with demographic characteristics between CHD or CHD-T2DM patients and control participants.

\begin{tabular}{lccc}
\hline Characteristic & OR $(95 \%$ CI $)$ & $P$ value & CHD-T2DM \\
\hline Age & $1.066(1.046-1.088)$ & 0.110 & $1.083(0.963-1.203)$ \\
Male & $1.413(0.911-2.190)$ & 0.122 & $1.383(1.160-1.606)$ \\
HDL & $0.885(0.436-1.795)$ & 0.735 & $0.276(0.119-0.635)$ \\
LDL & $1.126(0.827-1.534)$ & 0.450 & $0.958(0.705-1.302)$ \\
Total cholesterol & $1.060(0.867-1.295)$ & 0.569 & $0.915(0.747-1.120)$ \\
Triglyceride & $1.073(0.859-1.340)$ & 0.534 & $1.366(1.088-1.716)$ \\
Lipoprotein (a) & $0.670(0.263-1.709)$ & 0.402 & $0.253(0.084-0.764)$ \\
Lipoprotein (b) & $1.438(0.706-2.927)$ & 0.317 & $1.456(0.768-2.759)$ \\
Blood glucose & $1.001(0.865-1.158)$ & 0.993 & $2.388(1.874-3.043)$ \\
hs-TnI & $1.737(0.283-10.06)$ & 0.371 & $1.724(0.001-8.053)$ \\
CKMB & $1.446(1.044-2.002)$ & 0.026 & $1.867(1.305-2.672)$ \\
TMAO & $3.621(2.581-5.080)$ & 0.001 & $9.581(5.451-16.840)$ \\
\hline
\end{tabular}

TABLE 3: Multivariate regression analysis for the association of TMAO with demographic characteristics between CHD or CHD-T2DM patients and control participants.

\begin{tabular}{|c|c|c|c|c|}
\hline \multirow{2}{*}{ Characteristic } & \multicolumn{2}{|c|}{$\mathrm{CHD}$} & \multicolumn{2}{|c|}{ CHD-T2DM } \\
\hline & OR (95\% CI) & $P$ value & OR (95\% CI) & $P$ value \\
\hline HDL & $1.392(0.218-8.868)$ & 0.726 & $0.275(0.011-6.876)$ & 0.432 \\
\hline Triglyceride & $1.144(0.812-1.612)$ & 0.434 & $1.196(0.765-1.870)$ & 0.434 \\
\hline Lipoprotein (a) & $0.586(0.045-7.657)$ & 0.682 & $1.357(1.001-2.134)$ & 0.558 \\
\hline hs-TnI & $2.599(0.183-3.696)$ & 0.481 & $1.290(1.001-1.831)$ & 0.285 \\
\hline CKMB & $1.427(0.994-2.048)$ & 0.054 & $1.250(0.742-2.106)$ & 0.403 \\
\hline TMAO & $3.469(2.294-5.245)$ & 0.001 & $9.110(4.427-18.750)$ & 0.001 \\
\hline
\end{tabular}

TABLE 4: Correlation analysis for the association of TMAO with clinical parameters in $\mathrm{CHD}$ and CHD-T2DM patients.

\begin{tabular}{lcccc}
\hline \multirow{2}{*}{$\begin{array}{l}\text { Cardiovascular risk } \\
\text { factors }\end{array}$} & \multicolumn{4}{c}{ CHD } \\
Coefficient & $P$ value & Coefficient & $P$ value \\
\hline HDL & 0.174 & 0.001 & 0.167 & 0.006 \\
LDL & 0.009 & 0.865 & 0.006 & 0.920 \\
Total cholesterol & 0.145 & 0.006 & 0.018 & 0.759 \\
Triglyceride & 0.038 & 0.472 & 0.182 & 0.002 \\
Lipoprotein (a) & 0.034 & 0.525 & 0.109 & 0.072 \\
Lipoprotein (b) & 0.189 & 0.001 & 0.080 & 0.186 \\
Blood glucose & 0.031 & 0.564 & 0.176 & 0.003 \\
hs-TnI & 0.127 & 0.052 & 0.221 & 0.001 \\
CKMB & 0.125 & 0.034 & 0.216 & 0.001 \\
\hline
\end{tabular}

coronary syndromes [23]. Meantime, a clinical study exploring the prognostic value of TMAO in patients indicated that the strength of the association between TMAO and adverse events differed somewhat by race, with whites showing a significant association between elevated TMAO levels and heightened incident risk of cardiac death and any-cause death, whereas in blacks, higher TMAO levels were only significantly associated with cardiac death [24]. Our results in Chinese are consistent with these other clinical studies in that they show elevated levels of TMAO in cardiovascular disease. However, to determine if TMAO may serve as an independent risk factor for long-term mortality, further study is needed in the future.

TMAO induced cardiovascular disease via a multiplicity of effects, including changes in macrophage and endothelial dysfunction induced by vascular oxidative stress and inflammation [25-27]. In addition, TMAO has an effect on platelet activation, inducing platelet hyperreactivity leading to a prothrombotic effect and promoting thrombus formation [8]. All these effects are important mechanisms of TMAOinduced CHD. In our study, we found that the levels of TMAO were remarkably increased in CHD patients with T2DM compared with CHD patients. The result was consistent with that of the previous study $[14,28]$. TMAO has also been suggested to be a strong candidate molecule to mediate the development of T2DM in animal and human studies [29]. On the other hand, T2DM patients presented with an alteration in gut microbiota equilibrium, a disruption of gut barrier function, and an increase in gut permeability which altogether might result in aberrant production and absorption of TMAO [28]. The high levels of TMAO induced by T2DM could be influencing the progression of CHD through 
alterations in cholesterol and bile acid metabolism, activation of inflammatory pathways, and promotion of foam cell formation. This situation will increase the risk of major adverse cardiovascular events and death in the CHD-T2DM patients.

Furthermore, the previous study result suggested that decreasing the plasma levels of TMAO by selectively inhibiting microbial trimethylamine (TMA) production with a drug could serve as a means of inhibiting diet-induced atherosclerosis in animal models [30]. Similarly, the suppression of TMAO with approaches to knock down flavin monooxygenase 3 (the major hepatic enzyme responsible for the conversion of microbial-generated TMA into TMAO) could also inhibit atherosclerosis in animal models [31-33]. Thus, these results indicated that TMAO not only appeared to be a risk marker but also might serve as a potential therapeutic target to treat disease, including patients with $\mathrm{CHD}$ or $\mathrm{CHD}$ T2DM. Therefore, we speculate that rapid and accurate determination of TMAO levels could significantly improve rapid diagnosis and risk stratification between patients presenting with CHD and CHD-T2DM, which could improve the effect of treatment on the disease. At present, TMAO is known to be a plasma biomarker in patients at high risk for the development of T2DM and advanced chronic kidney disease [34]. In our study, we suggest that circulating TMAO may be a risk factor in T2DM-induced CHD, but the study points to TMAO as a potential therapeutic target for the clinical management of T2DM-induced CHD, which needs further study.

\section{Conclusions}

TMAO was an independent predictor of CHD; moreover, the TMAO levels were highly associated with diabetes in Chinese CHD patients, which could potentially refine CHD stratification in diabetes patients. The importance of our findings is underlined by the modifiable nature of TMAO, both with diet and with potential therapeutics under development, offering new prospects in treatment strategies for Chinese CHD patients with or without T2DM.

\section{Data Availability}

The data (determination of TMAO by LC-MS/MS) used to support the findings of this study are included within the article.

\section{Conflicts of Interest}

The authors have no conflicting financial interests.

\section{Authors' Contributions}

Zengxiang Dong, Zhaoguang Liang, Meihua Guo, Shuang $\mathrm{Hu}$, and Zhaoqian Shen collected the blood samples, performed the study, and supervised all the aspects of the research and analysis. Xin Hai was responsible for the design of the study and reviewed the manuscript. All authors read and approved the final manuscript. Zengxiang Dong and Zhaoguang Liang contributed equally to this article.

\section{Acknowledgments}

This work was supported by the National Natural Science Foundation of China (nos. 81400250 and 81700151).

\section{References}

[1] R. H. Eckel, M. Wassef, A. Chait et al., "Prevention conference VI: diabetes and cardiovascular disease: writing group II: pathogenesis of atherosclerosis in diabetes," Circulation, vol. 105, no. 18, pp. 138e-1143, 2002

[2] P. Severino, A. D’Amato, L. Netti et al., "Diabetes mellitus and ischemic heart disease: the role of ion channels," International Journal of Molecular Sciences, vol. 19, no. 3, p. 802, 2018.

[3] P. Y. Wu, C. L. Huang, W. S. Lei, and S. H. Yang, "Alternative health eating index and the dietary guidelines from American Diabetes Association both may reduce the risk of cardiovascular disease in type 2 diabetes patients," Journal of Human Nutrition and Dietetics, vol. 29, no. 3, pp. 363-373, 2016.

[4] P. L. Zock, W. A. M. Blom, J. A. Nettleton, and G. Hornstra, "Progressing insights into the role of dietary fats in the prevention of cardiovascular disease," Current Cardiology Reports, vol. 18, no. 11, p. 111, 2016.

[5] Z. Wang, E. Klipfell, B. J. Bennett et al., "Gut flora metabolism of phosphatidylcholine promotes cardiovascular disease," Nature, vol. 472, no. 7341, pp. 57-63, 2011.

[6] W. H. W. Tang, Z. Wang, B. S. Levison et al., "Intestinal microbial metabolism of phosphatidylcholine and cardiovascular risk," The New England Journal of Medicine, vol. 368, no. 17, pp. 1575-1584, 2013.

[7] R. A. Koeth, Z. Wang, B. S. Levison et al., "Intestinal microbiota metabolism of L-carnitine, a nutrient in red meat, promotes atherosclerosis," Nature Medicine, vol. 19, no. 5, pp. 576-585, 2013.

[8] W. Zhu, J. C. Gregory, E. Org et al., "Gut microbial metabolite TMAO enhances platelet hyperreactivity and thrombosis risk," Cell, vol. 165, no. 1, pp. 111-124, 2016.

[9] Z. Wang, W. H. W. Tang, J. A. Buffa et al., "Prognostic value of choline and betaine depends on intestinal microbiotagenerated metabolite trimethylamine-N-oxide," European Heart Journal, vol. 35, no. 14, pp. 904-910, 2014.

[10] W. H. W. Tang, Z. Wang, Y. Fan et al., "Prognostic value of elevated levels of intestinal microbe-generated metabolite trimethylamine-N-oxide in patients with heart failure: refining the gut hypothesis," Journal of the American College of Cardiology, vol. 64, no. 18, pp. 1908-1914, 2014.

[11] W. H. W. Tang, Z. Wang, K. Shrestha et al., "Intestinal microbiota-dependent phosphatidylcholine metabolites, diastolic dysfunction, and adverse clinical outcomes in chronic systolic heart failure," Journal of Cardiac Failure, vol. 21, no. 2, pp. 91-96, 2015.

[12] T. Suzuki, L. M. Heaney, S. S. Bhandari, D. J. L. Jones, and L. L. $\mathrm{Ng}$, "Trimethylamine $\mathrm{N}$-oxide and prognosis in acute heart failure," Heart, vol. 102, no. 11, pp. 841-848, 2016.

[13] E. Randrianarisoa, A. Lehn-Stefan, X. Wang et al., "Relationship of serum trimethylamine $\mathrm{N}$-oxide (TMAO) levels with early atherosclerosis in humans," Scientific Reports, vol. 6, no. 1 , article $26745,2016$.

[14] M. Dambrova, G. Latkovskis, J. Kuka et al., "Diabetes is associated with higher trimethylamine $\mathrm{N}$-oxide plasma levels," Experimental and Clinical Endocrinology \& Diabetes, vol. 124, no. 4, pp. 251-256, 2016. 
[15] V. Senthong, X. S. Li, T. Hudec et al., "Plasma trimethylamine $\mathrm{N}$-oxide, a gut microbe-generated phosphatidylcholine metabolite, is associated with atherosclerotic burden," Journal of the American College of Cardiology, vol. 67, no. 22, pp. 26202628, 2016.

[16] V. Senthong, Z. Wang, X. S. Li et al., "Intestinal microbiotagenerated metabolite trimethylamine-N-oxide and 5-year mortality risk in stable coronary artery disease: the contributory role of intestinal microbiota in a COURAGE-like patient cohort," J. Am. Heart. Assoc., vol. 5, no. 6, article e002816, 2016.

[17] X. Hai, V. Landeras, M. A. Dobre, P. DeOreo, T. W. Meyer, and T. H. Hostetter, "Mechanism of prominent trimethylamine oxide (TMAO) accumulation in hemodialysis patients," PLoS One, vol. 10, no. 12, article e0143731, 2015.

[18] N. Townsend, M. Nichols, P. Scarborough, and M. Rayner, "Cardiovascular disease in Europe 2015: epidemiological update," European Heart Journal, vol. 36, no. 40, pp. 26732674, 2015.

[19] R. Vedanthan, B. Seligman, and V. Fuster, "Global perspective on acute coronary syndrome: a burden on the young and poor," Circulation Research, vol. 114, no. 12, pp. 1959-1975, 2014.

[20] J. M. Oliver, P. Gallego, A. E. Gonzalez et al., "Impact of age and sex on survival and causes of death in adults with congenital heart disease," International Journal of Cardiology, vol. 245, pp. 119-124, 2017.

[21] T. Yamashita, K. Kasahara, T. Emoto et al., "Intestinal immunity and gut microbiota as therapeutic targets for preventing atherosclerotic cardiovascular diseases," Circulation Journal, vol. 79, no. 9, pp. 1882-1890, 2015.

[22] E. Org, M. Mehrabian, and A. J. Lusis, "Unraveling the environmental and genetic interactions in atherosclerosis: central role of the gut microbiota," Atherosclerosis, vol. 241, no. 2, pp. 387-399, 2015.

[23] X. S. Li, S. Obeid, R. Klingenberg et al., "Gut microbiotadependent trimethylamine N-oxide in acute coronary syndromes: a prognostic marker for incident cardiovascular events beyond traditional risk factors," European Heart Journal, vol. 38, no. 11, pp. 814-824, 2017.

[24] T. Shafi, N. R. Powe, T. W. Meyer et al., "Trimethylamine $\mathrm{N}$-oxide and cardiovascular events in hemodialysis patients," Journal of the American Society of Nephrology, vol. 28, no. 1, pp. 321-331, 2017.

[25] J. Geng, C. Yang, B. Wang et al., "Trimethylamine N-oxide promotes atherosclerosis via CD36-dependent MAPK/JNK pathway," Biomedicine \& Pharmacotherapy, vol. 97, pp. 941947, 2018.

[26] K. M. Boini, T. Hussain, P. L. Li, and S. S. Koka, "Trimethylamine-N-oxide instigates NLRP3 inflammasome activation and endothelial dysfunction," Cellular Physiology and Biochemistry, vol. 44, no. 1, pp. 152-162, 2018.

[27] T. Li, C. Gua, B. Wu, and Y. Chen, "Increased circulating trimethylamine N-oxide contributes to endothelial dysfunction in a rat model of chronic kidney disease," Biochemical and Biophysical Research Communications, vol. 495, no. 2, pp. 2071-2077, 2018.

[28] L. Sanchez-Alcoholado, D. Castellano-Castillo, L. JordánMartínez et al., "Role of gut microbiota on cardio-metabolic parameters and immunity in coronary artery disease patients with and without type-2 diabetes mellitus," Frontiers in Microbiology, vol. 8, p. 1936, 2017.
[29] Y. Kim, J. Keogh, and P. Clifton, "A review of potential metabolic etiologies of the observed association between red meat consumption and development of type 2 diabetes mellitus," Metabolism, vol. 64, no. 7, pp. 768-779, 2015.

[30] Z. Wang, A. B. Roberts, J. A. Buffa et al., "Non-lethal inhibition of gut microbial trimethylamine production for the treatment of atherosclerosis," Cell, vol. 163, no. 7, pp. 1585-1595, 2015.

[31] J. Miao, A. V. Ling, P. V. Manthena et al., "Flavin-containing monooxygenase 3 as a potential player in diabetes-associated atherosclerosis," Nature Communications, vol. 6, no. 1, p. 6498, 2015.

[32] D. M. Shih, Z. Wang, R. Lee et al., "Flavin containing monooxygenase 3 exerts broad effects on glucose and lipid metabolism and atherosclerosis," Journal of Lipid Research, vol. 56, no. 1, pp. 22-37, 2015.

[33] M. Warrier, D. M. Shih, A. C. Burrows et al., "The TMAOgenerating enzyme flavin monooxygenase 3 is a central regulator of cholesterol balance," Cell Reports, vol. 10, no. 3, pp. 326-338, 2015.

[34] M. A. I. Al-Obaide, R. Singh, P. Datta et al., "Gut microbiotadependent trimethylamine- $N$-oxide and serum biomarkers in patients with T2DM and advanced CKD," Journal of Clinical Medicine, vol. 6, no. 9, p. 86, 2017. 


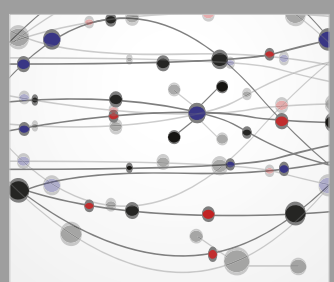

The Scientific World Journal
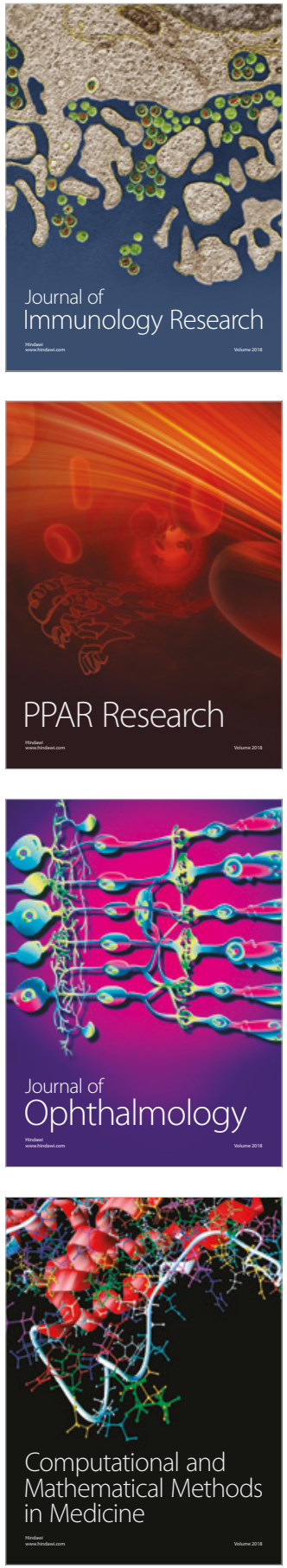

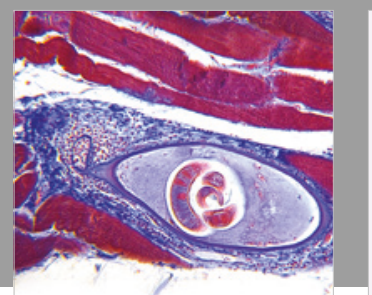

Gastroenterology Research and Practice

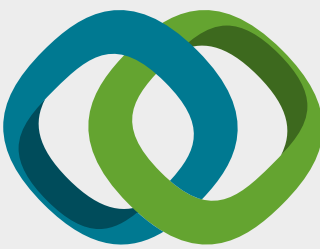

\section{Hindawi}

Submit your manuscripts at

www.hindawi.com
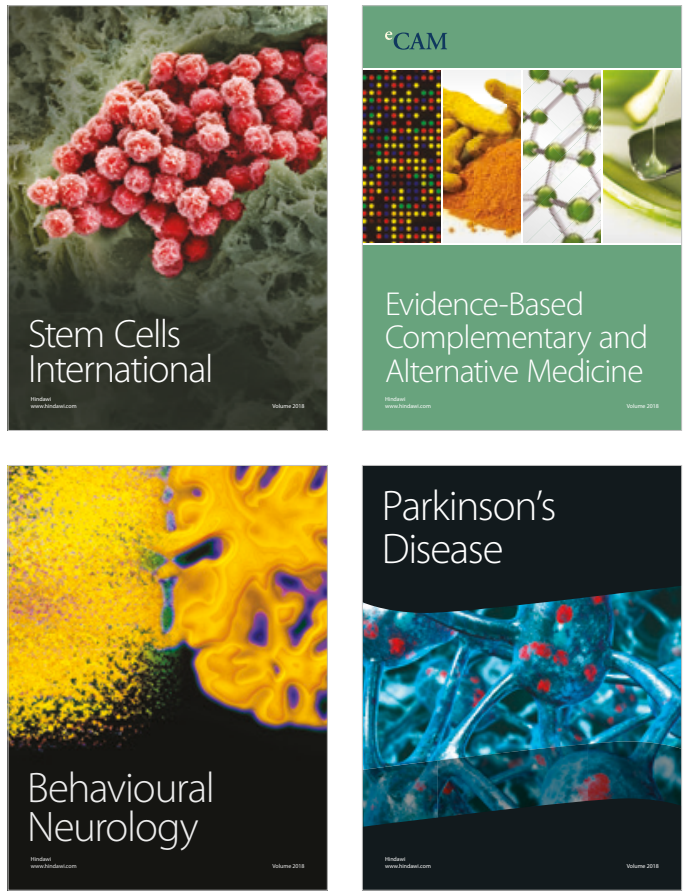

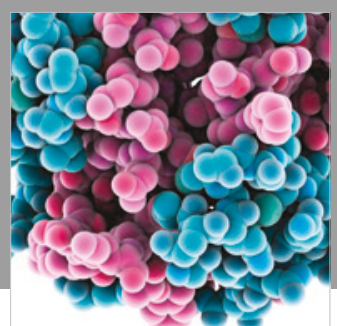

ournal of

Diabetes Research

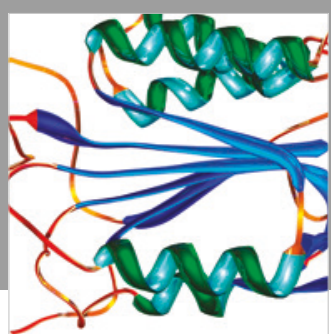

Disease Markers
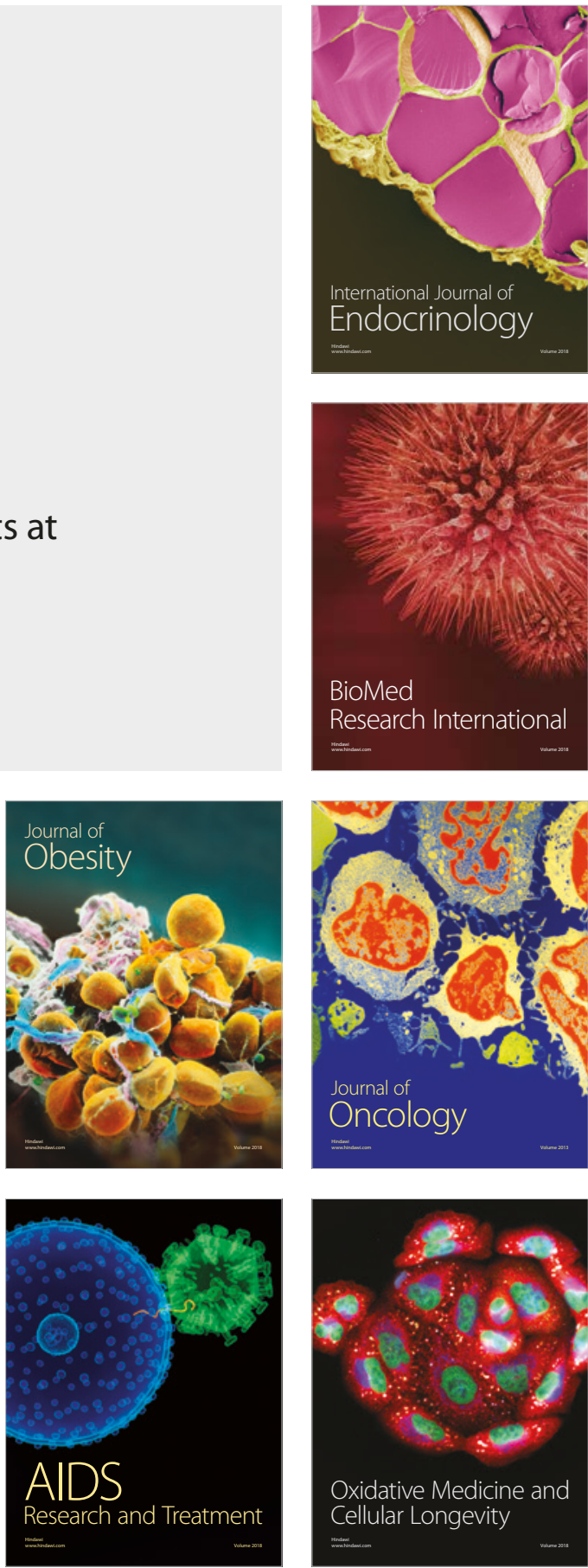